\title{
Ironing out mechanisms of iron homeostasis and disorders of iron deficiency
}

\author{
Navid Koleini, Jason S. Shapiro, Justin Geier, and Hossein Ardehali \\ Feinberg Cardiovascular Research Institute, Northwestern University, Chicago, Illinois, USA
}

\begin{abstract}
Iron plays an important role in mammalian physiological processes. It is a critical component for the function of many proteins, including enzymes that require heme and iron-sulfur clusters. However, excess iron is also detrimental because of its ability to catalyze the formation of reactive oxygen species. As a result, cellular and systemic iron levels are tightly regulated to prevent oxidative damage. Iron deficiency can lead to a number of pathological conditions, the most prominent being anemia. Iron deficiency should be corrected to improve adult patients' symptoms and to facilitate normal growth during fetal development and childhood. However, inappropriate use of intravenous iron in chronic conditions, such as cancer and heart failure, in the absence of clear iron deficiency can lead to unwanted side effects. Thus, this form of therapy should be reserved for certain patients who cannot tolerate oral iron and need rapid iron replenishment. Here, we will review cellular and systemic iron homeostasis and will discuss complications of iron deficiency.
\end{abstract}

\section{Introduction}

Iron is among the most abundant elements on Earth and is found naturally in two oxidation states - ferric iron $\left(\mathrm{Fe}^{3+}\right)$ and, more scarcely, ferrous iron $\left(\mathrm{Fe}^{2+}\right)(1)$. Mammalian cells primarily use ferrous iron, and its paucity on Earth resulted in the development of evolutionary mechanisms to efficiently uptake the oxidized form into the cell through its solubilization by environment acidification, followed by reduction to ferrous iron and its cellular transportation (1). Owing to its unique oxidation-reduction properties, iron can readily donate and accept electrons from various substrates, rendering it an important cofactor in biological processes. Additionally, iron is essential for heme and iron-sulfur (Fe-S) clusters and has various functions independent of its oxygen-binding capacity, including demethylation, dehydrogenation, and reduction of sulfur (2-4).

The iron content of an average adult human is estimated to be about 3-4 g, of which 2-3 g is bound within iron-rich hemoglobin in red blood cells (RBCs) (5). Although many cellular enzymes and proteins require iron for proper functioning, excessive iron is associated with oxidative stress and cellular damage (6). The mechanism of iron toxicity is rooted in iron's ability to donate electrons to an oxygen-containing molecule to generate hydroxyl radicals via the Fenton reaction (6). Hydroxyl radicals are extremely toxic reactive oxygen species (ROS) molecules and can damage cellular components such as lipids, DNA, and proteins. Because of this toxic effect of iron, its cellular levels are tightly regulated.

\section{Systemic iron homeostasis}

Systemic iron absorption

Iron can be absorbed in the intestinal tract as both heme and non-heme iron (7). Compared with non-heme iron, heme iron

Conflict of interest: Hossein Ardehali receives income as an expert witness. Copyright: (c) 2021, American Society for Clinical Investigation.

Reference information: J Clin Invest. 2021;131(11):e148671.

https://doi.org/10.1172/JCl148671. has superior bioavailability. Although the mechanism of heme absorption by enterocytes is not well known, heme carrier protein 1 (HCP1, whose primary function is to transport folate; ref. 8) has been suggested as a candidate mediating the influx of heme in enterocytes (9). Upon uptake into enterocytes, heme oxygenase-1 (HO-1) mediates the extraction of ferrous iron from heme and its subsequent release into the cytoplasm (Figure $1 \mathrm{~A}$ and ref. 10).

For non-heme iron absorption, reduction of ferric to ferrous iron is facilitated at the brush border of the gut lumen through several mechanisms, including the low-pH environment of the proximal intestinal lumen (11) and the action of ferrireductases, including duodenal cytochrome $b$ reductase 1 (DCYTB) (Figure $1 \mathrm{~B}$ and ref. 12). After iron's reduction to the ferrous form, divalent metal transporter 1 (DMT1) in duodenal and proximal jejunal enterocytes mediates iron absorption (13). DMT1's critical role in regulating intestinal iron absorption is evidenced by decreased iron absorption in mice with intestine-specific DMT1 deletion (14) and the finding that the most prevalent form of hemochromatosis is associated with upregulation of DMT1 (15).

Once iron is imported into enterocytes (or released within macrophages, as described below), it can be stored by binding to ferritin (FTN) or exported into the circulation through the only iron exporter, ferroportin-1 (FPN1), which is present on the basolateral surface of enterocytes (Figure 1, A and B, and refs. 16, 17). Total-body stores of iron determine whether iron is transported into the circulation or remains in enterocytes. When body iron levels are low, iron is exported into the circulation by FPN1 and immediately oxidized to ferric iron by enterocyte copper-containing ferroxidases, including ceruloplasmin and hephaestin $(16,18,19)$. In the blood, ferric iron has a high affinity for the soluble serum protein transferrin (TF), the main carrier of iron for distribution throughout the body (20). When body iron levels are high, the liver secretes hepcidin (discussed below), which binds to FPN1 and leads to its degradation or 


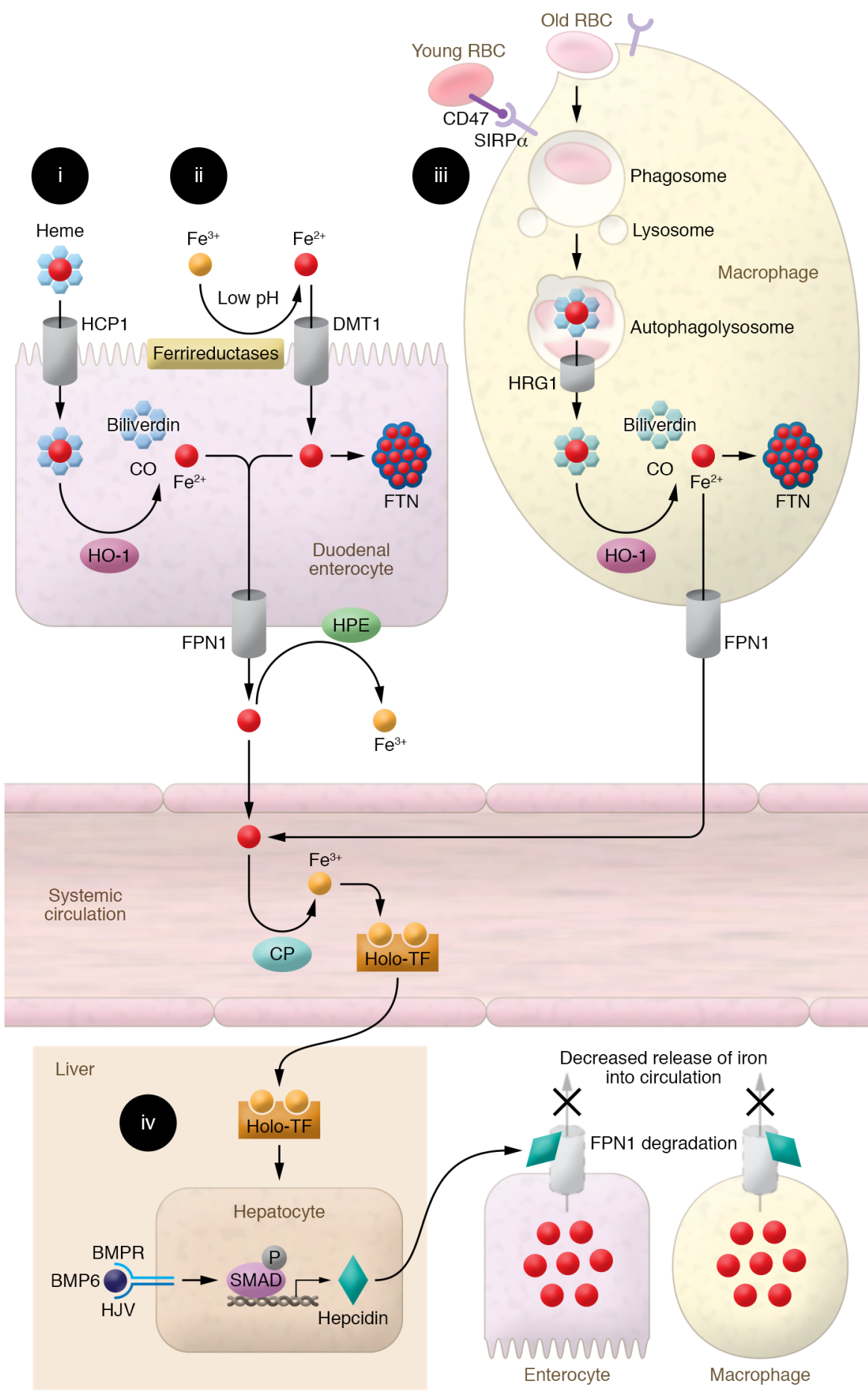

Figure 1. Depiction of the mechanism of systemic iron regulation. (i) Heme-bound iron is absorbed into duodenal enterocytes, possibly via $\mathrm{HCP} 1$. Enterocyte $\mathrm{HO}-1$ releases iron from heme's porphyrin ring, producing $\mathrm{Fe}^{2+}$, biliverdin, and carbon monoxide. $\mathrm{Fe}^{2+}$ iron is then exported into the circulation by FPN1. (ii) Absorbed $\mathrm{Fe}^{3+}$ is reduced to $\mathrm{Fe}^{2+}$ at the brush border by low $\mathrm{pH}$ and ferrireductases, enabling its transport by DMT1 into duodenal enterocytes. $\mathrm{Fe}^{2+}$ is either bound to FTN within the enterocyte, limiting the intracellular pool of free iron, or released into the circulation by FPN1, where it is oxidized to $\mathrm{Fe}^{3+}$ by hephaestin (HPE) and ceruloplasmin (CP) and bound to TF for transport. (iii) Macrophages identify senescent RBCs no longer expressing CD47 via SIRP $\alpha$ and recycle RBC iron through phagocytosis. Upon fusion of phagosomes with lysosomes, heme is released from hemoglobin and transported to the cytosol via HRG1. In the cytosol, heme-bound iron is extracted by $\mathrm{HO}-1$ and exported by FPN1. $\mathrm{Fe}^{2+}$ is then oxidized to $\mathrm{Fe}^{3+}$ by $\mathrm{CP}$ and binds to TF for transport. (iv) When systemic iron levels are sufficiently replete, hepcidin is produced by the liver, binds to FPN1 on macrophages and enterocytes, and promotes its degradation. This prevents intestinal and macrophage iron release into the circulation. Binding of BMP6 to BMP receptor and its coreceptor HJV activates SMAD signaling and promotes transcription of hepcidin.

\section{Iron recycling by macrophages}

The daily iron requirement in a human adult is about $25 \mathrm{mg}$. However, intestinal absorption capacity usually does not exceed 1-2 mg of iron per day. The remaining iron supply comes from recycling of iron in senescent cells - particularly old RBCs (Figure 1C). Young RBCs express CD47 on their surface, presenting a "do not eat me" signal recognized by signal regulatory protein- $\alpha(\operatorname{SIRP} \alpha)$ on the macrophage surface. In contrast, senescent RBCs lose CD47 and are rapidly cleared from the circulation by macrophages in the reticuloendothelial system (23-25). The heme prosthetic group gets released from the globin protein in the acidic phagolysosome compartment in macrophages and is exported into the cyto-

occludes iron efflux (21). As a result, iron is trapped inside the duodenal enterocytes, and since these cells have a lifespan of only 3-4 days, their sequestered iron content is excreted when they are eventually sloughed (22).

Although there is a sophisticated system in place to ensure coordinated uptake of iron into the body, mammals have no system to excrete excess iron (except for acute blood loss and phlebotomy) (24), suggesting that there was likely an evolutionary pressure on organisms to uptake and conserve scarce environmental iron to avoid iron deficiency (ID).

plasm via the heme transporter HRG1 (26). HO-1 then releases iron from heme and metabolizes the iron-free heme into biliverdin and carbon monoxide.

\section{Systemic iron regulation}

Systemic iron levels are mainly regulated by hypoxia, inflammation, and hepcidin (which is itself regulated by hypoxia and inflammation; refs. 27, 28).

Regulation of systemic iron by hepcidin. Hepcidin antimicrobial peptide (HAMP1) is a small (25-amino acid) hormone peptide 
secreted by hepatocytes in conditions of iron sufficiency. It binds to FPN1, promoting its internalization and degradation, resulting in the inhibition of iron absorption from the digestive tract and release of iron from macrophages (Figure 1D and ref. 29). Several cellular pathways are involved in regulating hepcidin in the liver, including normal/high iron levels, inflammation, erythropoietic signaling, and hypoxia. In conditions of normal/high iron, BMP6 binds to BMP receptor (BMPR) and its coreceptor, hemojuvelin (HJV), activating the BMP6/HJV/BMPR complex on the surface of hepatocytes and subsequent downstream signaling to SMAD1/5/8 (Figure 1D and refs. 30, 31). Recent evidence suggests that endothelial cells are the major source of BMP6 in the liver, and regulate hepcidin production in hepatocytes through a paracrine effect (32). In addition to BMP6, endothelial cell BMP2 also plays a role in hepcidin regulation by iron (33). Additionally, a recent paper showed that NRF2 controls iron homeostasis in hemochromatosis and thalassemia through BMP6 and hepcidin (34). Finally, hepcidin production is inhibited in response to erythroferrone (ERFE), which is secreted from erythroblasts when hematopoiesis is induced in response to erythropoietin (EPO). This regulation of hepcidin by ERFE results in an increased availability of circulatory iron for efficient erythropoiesis $(35,36)$. The activated SMADs directly bind to $\mathrm{BMP}$ response elements located in the HAMP1 promoter, thereby upregulating its transcription (37). Mutations in HJV (G320V being most common), TF receptor-2 (TFR2), HAMP1, FPN1, and the homeostatic iron regulator HFE cause hereditary hemochromatosis (a disease of iron overload manifested by cirrhosis, cardiomyopathy, skin darkening, diabetes, and possibly death) (38-40).

Regulation of systemic iron by hypoxia. Systemic iron can also be regulated by oxygen through hypoxia-inducible factor-1 (HIF1) and HIF2. Anemia can cause hypoxia within vital tissues, inactivating cytoplasmic prolyl hydroxylase domain proteins (PHDs) whose activity requires iron as a cofactor (41). Under conditions of normoxia, PHDs promote protein hydroxylation of HIF1/2, leading to the recognition of HIF1/2 by von Hippel-Lindau tumor suppressor (VHL) protein and polyubiquitination/proteasomal degradation of HIF. When $\mathrm{O}_{2}$ tension drops, PHD enzymes are inactivated and HIF1 and HIF2 become stable and translocate into the nucleus. Nuclear translocation of HIF $2 \alpha$ results in the activation of its target genes, including the iron uptake genes TFR1 and $D M T 1$ in enterocytes, as well as the erythropoietic stimulatory hormone EPO in the kidney $(42,43)$. This coordinated mechanism results in increased systemic iron uptake and RBC production.

Regulation of systemic iron by inflammation. Bacteria require sufficient quantities of iron to proliferate and establish infection. This is accomplished through secretion of specialized iron-sequestering proteins called siderophores, which bind iron in serum to facilitate its uptake by the invading bacteria. However, mammalian hosts have developed systems to withhold their iron from bacteria through secretion of lactoferrin, haptoglobin, and certain inflammatory signals, which induce hepcidin production $(44,45)$. The overproduction of hepcidin in inflammation and subsequent FPN1 degradation inhibit iron release from the reticuloendothelial system and duodenal enterocytes, resulting in limited iron availability for erythropoiesis and reduced RBC production in the bone marrow. Thus, although inflammation causes iron-starved bacteria, it can also lead to iron restriction in the host.
Iron restriction in the setting of inflammation can lead to anemia, known as anemia of chronic disease (ACD) (Figure 2A and ref. 29). This condition is characterized by decreased circulating iron levels, decreased TF saturation, and normal to increased FTN and iron storage levels (46). Iron supplementation or packed RBC transfusion is not indicated for the treatment of ACD with no concomitant ID. LDN193189, an inhibitor of SMAD proteins (which regulate the expression of hepcidin transcription), was shown to prevent the development of ACD in animal models (47). Hepcidin inhibitors and other strategies to increase FPN1 are also suggested as emerging therapies in ACD (48).

\section{Cellular iron homeostasis}

In addition to systemic iron homeostasis, cellular iron levels are also regulated by a sophisticated system involving iron-bound TF binding to its receptor (TFR1) (Figure 2B). The holo-TF complex contains two ferric iron molecules. TF/TFR1 binding causes clathrin-dependent endocytosis of the complete holo-complex, subsequent acidification of the endosome by vacuolar ATPase, and reduction of ferric to ferrous iron by the STEAP family of metalloreductases (25). This is followed by release of ferrous iron from the endosomes into the cytoplasm via DMT1 and shuttling of the apo-TF and TFR1 back to the cell surface for recycling (49).

In the cytoplasm, ferrous iron is oxidized to its ferric form by the bound FTN, whose levels are tightly correlated with total cellular iron content (Figure 2B and ref. 25). FTN-bound iron can be stored for later use or degraded and made available for enzymatic reactions. Iron-saturated FTN is degraded through nuclear receptor coactivator 4-mediated (NCOA4-mediated) autophagy, called ferritinophagy (Figure 2B and ref. 50). NCOA4 binding to FTN facilitates its delivery to pre-autophagosomes, which eventually fuse with lysosomes. Lysosomal degradation of FTN results in release of its iron content (51), which is then exported to the cytosol via lysosomal DMT1 (52).

\section{Mitochondrial iron regulation}

Mitochondria are the major site of heme and Fe-S cluster biosynthesis (53), so a large proportion of cellular iron is shuttled into the mitochondria. Iron uptake into the mitochondria is mediated by mitoferrin 1 (MFRN1; expressed mainly in cells of the erythroid lineage) and MRFN2 (ubiquitously expressed) (Figure 2C and ref. 54). Global knockout of MFRN1 results in embryonic lethality (55), and its knockout in hematopoietic stem cells causes severe anemia (54), while global MFRN2 knockout results in reduced sperm count and motility (56). Mitochondrial iron is sequestered by binding to mitochondria-specific ferritin (FTMT) to prevent ROS production. Mutations in FTMT cause mitochondrial iron overload and cytoplasmic ID, indicating that FTMT plays an active role in shuttling iron to the cytoplasm in addition to mitochondrial iron storage (57). The mechanism of mitochondrial iron export is not clear, but iron is likely exported from mitochondria either in the form of Fe-S clusters or heme or conjugated to glutathione (but not as free iron) (53). Our group has shown that deletion of the mitochondrial ATP-binding cassette $(\mathrm{ABC})$ protein $8 \mathrm{ABCB} 8$ leads to mitochondrial iron overload, ROS production, defects in cytosolic maturation of $\mathrm{Fe}-\mathrm{S}$ proteins, and cardiomyopathy (58). Similar functions were demonstrated for another mitochondrial ABC protein, $\mathrm{ABCB} 7$ (59). 
A

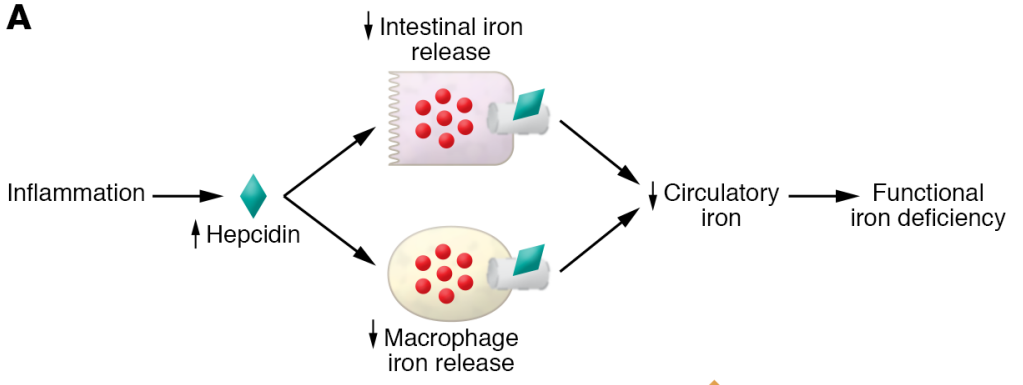

B

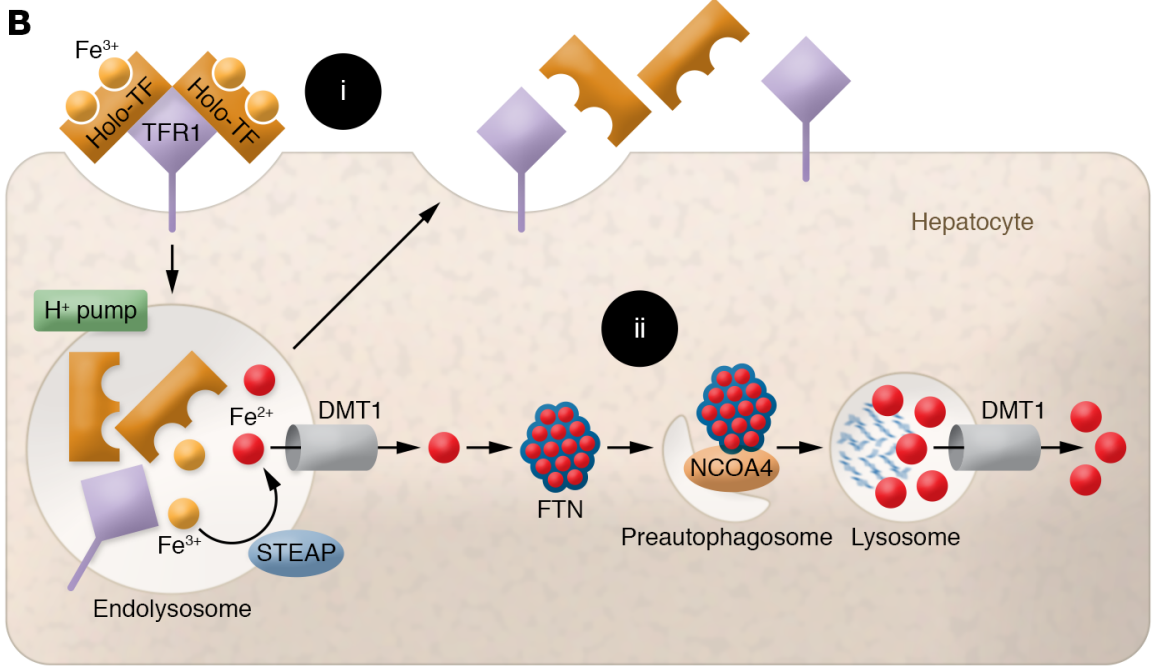

C

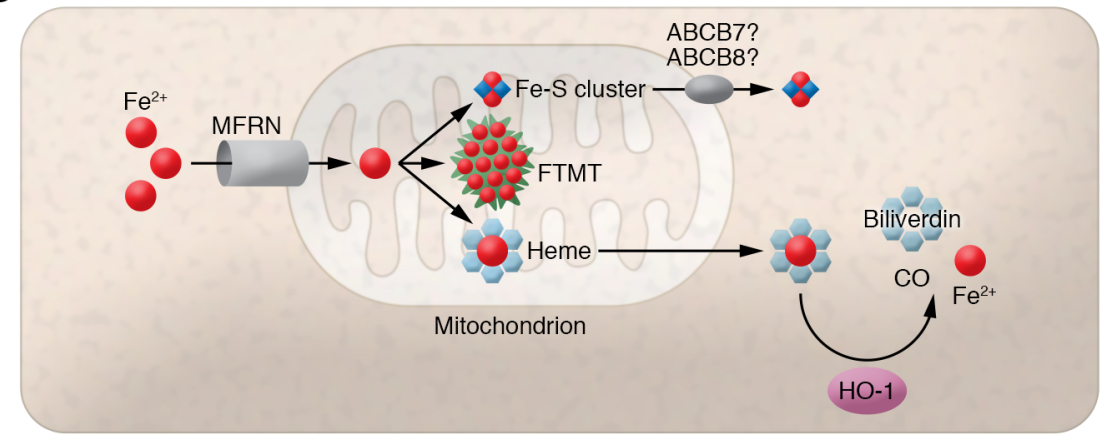

Figure 2. Depiction of the mechanism of ACD and cellular and mitochondrial iron regulation. (A) Graphic of pathways leading to functional iron deficiency (ID). Inflammation increases hepcidin expression, inhibiting intestinal and macrophage iron release into the circulation. This simultaneously decreases systemic iron bioavailability and traps iron within tissues, leading to functional ID despite normal iron storage. (B) (i) Iron bound to TF is absorbed by hepatocytes via binding to TFR1 and subsequent receptor-mediated endocytosis. Iron is reduced from $\mathrm{Fe}^{3+}$ to $\mathrm{Fe}^{2+}$ and released from TFR1-TF in acidified endolysosomes by the action of the STEAP family of ferrireductases. $\mathrm{Fe}^{2+}$ is then exported to the cytosol via DMT1, while TFR1 and TF are recycled back to the cell surface. (ii) Within the cytosol, iron is stored by binding to FTN to reduce free-iron toxicity. Iron is released from FTN via receptor (NCOA4)-mediated autophagy and is exported to the cytosol via lysosomal DMT1. (C) Elemental iron is imported across the mitochondrial membrane by MFRN1/2. Synthesis of Fe-S clusters and heme occurs primarily within mitochondria. Fe-S clusters are exported into the cytosol via unknown mechanisms, but may require $A B C B 7$ and $A B C B 8$.
Another ABC family member, ABCB10, regulates early steps of heme synthesis in the mitochondria, but it remains unclear what substrate it transports (60).

\section{Cellular iron regulation by IRE/IRP system}

The process of cellular iron regulation is mainly carried out post-transcriptionally by iron regulatory protein 1 (IRP1) and IRP2 (Figure 3A and refs. 61, 62). Binding of IRP1/2 to the $3^{\prime}$-UTR of a target transcript (such as TFR1) stabilizes the transcript and increases protein translation, while binding of IRP1/2 to the $5^{\prime}$-UTR (like that of FPN and FTN) sterically blocks ribosomal entry and prevents translation $(61,62)$ IRP1 is a bifunctional protein based on its binding to an Fe-S cluster. Holo-IRP1 functions as cytosolic aconitase and does not bind to mRNA; however, when iron levels are reduced, IRP1 becomes deprived of Fe-S clusters and the resultant apo-IRP1 binds to iron response elements (IREs). On the other hand, IRP2 is constitutively active, but under iron-sufficient conditions, it is ubiquitinated and proteolytically degraded in a process dependent on the protein FBXL5 $(61,62)$. Thus, when iron levels are normal, IRP1 regains its Fe-S cluster and IRP2 is degraded, resulting in inhibition of the IRP system (Figure 3A).

\section{Iron conservation by mTOR/TTP pathway}

During prolonged periods of severe ID, global iron availability is limited, and the body must ration and conserve this scarce element. Our laboratory has identified another major pathway that is engaged when the IRP system fails to maintain sufficient levels of iron during severe ID. This pathway is termed the iron conservation pathway, as opposed to the iron acquisition pathway mediated by the IRP system (Figure 3B and ref. 63). We have shown that in ID, an mRNA-binding protein called tristetraprolin (TTP) is induced, which paradoxically represses TFR1 expression and also leads to iron conservation inside the cell. TTP mediates degradation of target mRNAs via binding to CCR4-NOT transcription complex subunit 1 (CNOT1) of the deadenylase complex (64). ID-driven TTP induction is required for cell survival, 
A Low iron

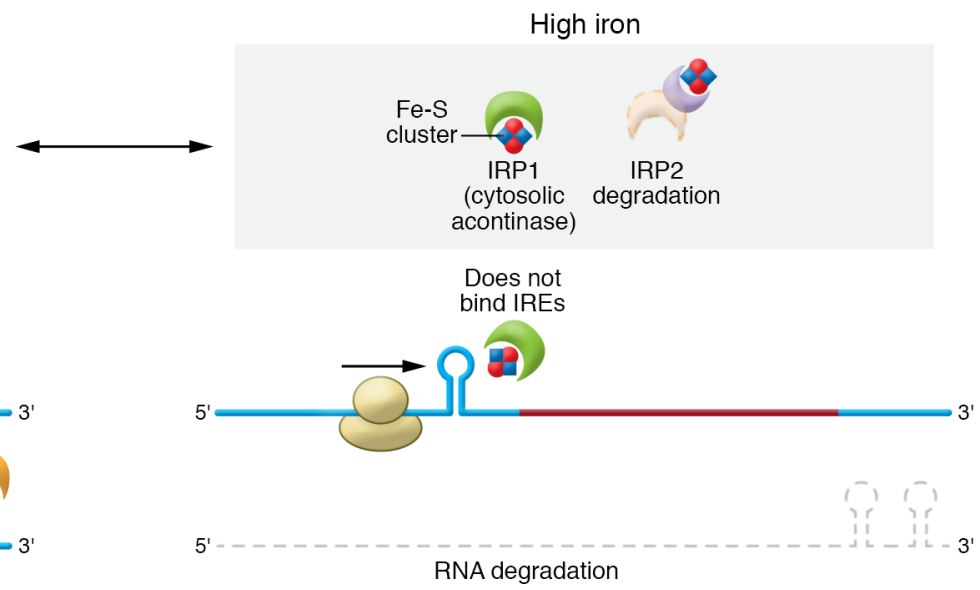

B Critically low iron

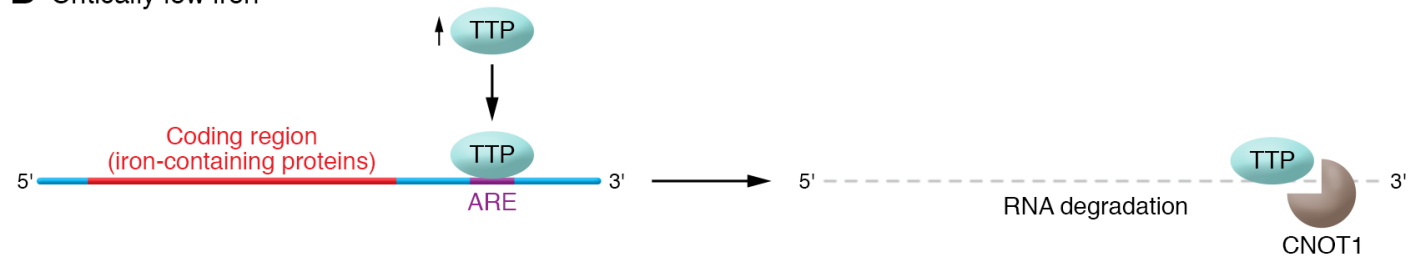

Figure 3. Regulation of cellular iron by the IRP system and TTP. (A) IRPs dynamically bind IREs in the UTRs of target mRNAs to regulate the expression of proteins important in iron metabolism. When iron is sufficient, IRP1 and IRP2 do not bind mRNA because (a) IRP1 binds Fe-S clusters, preventing IRP1 from binding to the IREs on target MRNAs, and (b) IRP2 is degraded in a process dependent on FBXL5. In ID, binding of IRPs to IREs located at the $5^{\prime}$-UTR of the transcripts causes steric blockage of ribosomal entry and prevention of translation (FPN, FTN). Binding of IRPs to IREs located on the 3'-UTR of target mRNAs increases stability of the transcript and thus increases translation (TFR1, DMT1). Accordingly, this leads to upregulated translation of iron acquisition proteins such as TFR1 and DMT1 and downregulation of proteins that bind or export iron such as FTN or FPN. (B) In severe ID, iron is preserved for vital processes via a mechanism called the iron conservation pathway. Critically low iron induces upregulation of TTP that binds AU-rich elements (AREs) in the 3'-UTR of target mRNAs. Under critically low iron conditions, ARE-bound TTP recruits CNOT1, a member of the CCR4-NOT deadenylase complex, to promote degradation of target mRNAs. Multiple TTP target mRNAs encode iron-binding proteins that could sponge the limited iron available in the cell, such as mitochondrial Fe-S-containing proteins, necessitating their translational downregulation in severe ID.

as TTP-knockout mice display cardiac dysfunction in response to ID (65), and TTP deletion in cultured cells results in increased cell death in low-iron conditions (63). After induction, TTP binds to and promotes the degradation of mRNA transcripts encoding proteins involved in a number of processes, including Fe-S clustercontaining proteins in the mitochondrial electron transport chain. When this process is activated during ID, excess apoproteins are not generated to sponge up iron, and limited iron is utilized only by the most essential proteins (65).

\section{Iron deficiency}

In this Review, we focus on ID and its pathological consequences, not other diseases of iron homeostasis including iron overload. In adults, ID generally leads to anemia, and other vital organs are protected. However, during fetal growth and childhood, when iron is critical for normal development, ID can lead to certain disorders.

\section{Absolute versus functional ID}

Absolute ID refers to depletion of total-body iron stores due to impaired nutrition, reduced absorption due to GI disease, use of proton pump inhibitors, and blood loss. Functional ID is associated with increased production of hepcidin in inflammatory con- ditions, causing reduced iron absorption from the gastrointestinal system and iron trapping in macrophages, which results in reduced circulatory iron levels (66). Serum FTN levels less than $15 \mathrm{ng} / \mathrm{mL}$ are highly specific for absolute ID; however, in inflammation, FTN levels increase and higher levels of FTN may occur with ID (67).

\section{Iron-deficiency anemia}

In mammals, the most common manifestation of ID is anemia, and in general, vital organs are protected from ID (except for the conditions described below). Iron-deficiency anemia (IDA) is defined as low hemoglobin or hematocrit associated with microcytic (low mean corpuscular volume) and hypochromic (low mean corpuscular hemoglobin) RBCs and low RBC count (67). IDA commonly affects women of reproductive age, children, patients with chronic diseases, and the elderly (68).

In ID, iron storage must become severely depleted before anemia occurs, since the recycling of iron from daily RBC turnover provides sufficient iron for erythropoiesis and hemoglobin production with modest reduction in iron stores (69). The body's response to IDA includes increased EPO secretion to stimulate erythropoiesis and decreased hepcidin production to increase intestinal iron uptake and mobilization of iron stores. 


\section{Fetal growth retardation}

IDA during pregnancy is associated with increased fetal and maternal complications, including low birth weight (LBW), preterm labor, smallness for gestational age (SGA), increased risk of maternal mortality, and risk of cesarean delivery. Additionally, increased risk of LBW, SGA, and preterm labor is correlated with the severity of IDA in the first trimester of pregnancy (70). The mechanisms by which ID causes fetal growth retardation are not well understood; however, attenuated insulin and insulin-like growth factor-1 (IGF-1) signaling in the maternal circulation has been proposed as a potential mechanism, as levels of these growth factors are reduced in ID $(71,72)$. Whether ID itself plays a direct role, independently of growth factor production, in fetal growth retardation is not known.

\section{Neurological deficits}

Brain development accelerates during the last trimester of pregnancy and requires sufficient amounts of iron to meet its highly elevated metabolic rate (73). In addition, neurogenesis and myelination require iron as a cofactor for cholesterol and lipid synthesis (74). ID, especially in the third trimester of pregnancy, has been shown to cause short-term and long-term neurological and cognitive deficits, as ID preterm infants show decreased central nerve conductance velocity and impaired neurological reflexes shortly after birth $(75,76)$. Infants exposed to ID during pregnancy also display delayed motor development and impaired recognition, auditory memory, and learning, as well as emotional and personality disorders, which persist even if ID is corrected after the critical period of brain development (76).

It is not clear which part of the brain is most affected by ID during development; however, animal studies suggest that ID impairs hippocampal development (77). Although mechanisms of hippocampal underdevelopment in ID are not well understood, it is known that ID can lead to impaired oxidative phosphorylation (77), epigenetic alterations $(78,79)$, and suppressed mTOR signaling $(80)$.

\section{Immune disorders}

ID has been shown to have a profound impact on vulnerability to infection, humoral response to vaccination, and cellular immunity. Children with ID are also more susceptible to developing upper respiratory tract and GI infections, which can be mitigated by iron supplementation $(81,82)$. Following vaccination, iron-deficient infants display lower plasma concentrations of pertussis, diphtheria, and pneumococcal immunoglobulins (83). Additionally, ID impairs activation of $\mathrm{T}$ lymphocytes $(84,85)$ and reduces the number of $\mathrm{CD}^{+}$and $\mathrm{CD} 8^{+} \mathrm{T}$ cells in the circulation (86), and these effects are improved by replenishing of iron stores (87). Finally, cell-mediated phagocytosis is attenuated in ID children, making them more vulnerable to salmonella infection (88).

There is no consensus on how ID impacts immunity; however, epigenetic alterations and post-transcriptional processes have been suggested as possible mechanisms. Epigenetic regulation of gene expression relies, in part, on a family of proteins containing Jumonji C (JmjC) domains, which use iron, $\alpha$-ketoglutarate, and oxygen to demethylate DNA and histones (89). In a mouse model of ID, enrichment of histone dimethyl marks at the cyclin E1 promoter was shown to reduce its promoter activity and reduce pro- liferation of B lymphocytes (90). Additionally, iron is required for stabilization of an mRNA-binding protein, Poly(rC)-binding protein-1 (PCBP1), whose binding to the 3 '-UTR of GM-CSF and IL-2 mRNAs increases their stability (91).

\section{Restless legs syndrome}

Restless legs syndrome (RLS), or Willis-Ekbom disease, affects $9 \%-15 \%$ of the adult population and is characterized by an irresistible urge to move the legs accompanied by an unpleasant sensation relieved by movement during rest or sleep (92). Although SNPs in a number of genes are associated with RLS $(93,94)$, RLS can also occur secondarily to brain ID (BID). BID's role in RLS is supported by findings showing decreased brain iron by magnetic resonance phase imaging, decreased cerebrospinal fluid FTN levels, and improvement of RLS symptoms by iron supplementation (95). The exact mechanism for RLS in BID is not well understood; however, impaired striatal dopamine production is widely seen in animal models of ID displaying symptoms characterized as RLS. Tyrosine hydroxylase, the rate-limiting enzyme in dopamine production, requires iron as a cofactor, and its activity is shown to be impaired in animal models of RLS due to BID (96). Although iron administration is not recommended empirically, trials of oral ferrous sulfate with vitamin $\mathrm{C}$ can be considered for RLS patients with FTN levels $\leq 75 \mathrm{ng} / \mathrm{mL}$ (97).

\section{Iron therapy for chronic diseases \\ Chronic kidney disease}

Normocytic anemia is a common finding among chronic kidney disease (CKD) patients, mainly due to elevated hepcidin (and reduced iron availability) and decreased EPO levels (98). Increased production of hepcidin is likely secondary to chronic inflammation and decreased renal clearance of this peptide (99). As a result of decreased renal production of EPO, CKD patients generally need erythropoiesis-stimulating agents (ESAs) (99). However, inefficient erythropoiesis following ESAs is frequently observed in CKD patients, likely due to decreased iron availability to the erythroid cell line (99).

As FTN levels are commonly higher in CKD patients as a result of chronic inflammation and uremia, absolute ID is defined as TF saturation (TSAT) less than $20 \%$ and FTN less than $100 \mathrm{ng} / \mathrm{mL}$ in predialysis and less than $200 \mathrm{ng} / \mathrm{mL}$ in dialysis patients $(100-$ 102), while anemia is defined as hemoglobin levels below $13 \mathrm{~g} / \mathrm{dL}$ in males and $12 \mathrm{~g} / \mathrm{dL}$ in females (103). While iron supplementation is strongly recommended in CKD patients with ID, there are subsets of patients with higher FTN levels who would also benefit from iron (103). Thus, Kidney Disease: Improving Global Outcomes (KDIGO) recommends one trial of $1 \mathrm{~g}$ i.v. iron or 1-3 months of $200 \mathrm{mg}$ oral iron per day in predialysis patients with TSAT less than $30 \%$ and FTN less than $500 \mathrm{ng} / \mathrm{mL}$, only if an increase in hemoglobin without starting ESA or an increase in hemoglobin and a decrease in ESA dose is desired (103).

Elevated hepcidin in CKD decreases intestinal iron absorption. Thus, oral iron supplementation may have limited efficacy, and i.v. iron administration is routinely recommended. Several clinical trials compared the efficacy and safety of i.v. versus oral iron formulation in CKD patients (98). A recent meta-analysis included 39 randomized and quasirandomized clinical trials with 
over 3800 patients comparing i.v. and oral iron supplementation in CKD (104) and concluded that there was insufficient evidence for superiority of either i.v. iron or oral iron in reducing mortality, slowing the time for initiation of dialysis, reducing blood transfusions, and improving quality of life. However, i.v. iron was superior in increasing hemoglobin, FTN, and TSAT and reducing the dose of ESAs (104). A reduction in the dose of ESAs is generally associated with beneficial effects, and it is interesting that this study did not show any outcome benefit with i.v. iron. In addition, i.v. iron was associated with increased allergic reactions and hypotension (104). Therefore, decisions on the route of iron delivery should be made with careful consideration in each patient. In CKD, especially in predialysis patients, i.v. iron administration should be limited to those with ID and severe anemia, those suffering from ongoing blood loss, and individuals with history of nonresponsiveness or side effects with oral iron formulations (103). Alternative strategies aiming at attenuation of inflammation in CKD patients would be a major step forward, as a recent study showed that a neutralizing IL-6 ligand antibody, ziltivekimab, decreases inflammation in CKD patients, attenuates ESA resistance, and increases serum iron and TSAT levels (105).

\section{Heart failure}

In heart failure (HF) patients, ID is defined as FTN less than 100 $\mathrm{ng} / \mathrm{mL}$, or FTN between 100 and $300 \mathrm{ng} / \mathrm{mL}$ and TSAT less than 20\%, as outlined in the 2017 American College of Cardiology (ACC)/American Heart Association (AHA) and the 2016 European Society of Cardiology (ESC) guidelines $(106,107)$. These criteria were adopted from the guidelines in CKD, although the mechanisms for these diseases are distinct. As discussed above, patients with CKD have elevated hepcidin; however, recent studies indicated that serum hepcidin levels are actually reduced in $\mathrm{HF}$ patients, and elevated hepcidin levels are not seen in anemic HF patients $(108,109)$. Thus, it is important to revisit these diagnostic criteria and design new criteria that identify patients with true ID.

The 2017 ACC/AHA and 2016 ESC guidelines recommend use of i.v. iron in HF patients with reduced ejection fraction (class IIa and IIb, respectively) $(106,107)$. A number of clinical trials have been conducted to evaluate i.v. iron supplementation in HF patients (110-112). A published meta-analysis of ten clinical trials on iron supplementation in HF patients revealed that iron administration was associated with improved cardiac function and quality of life and lower risk for hospitalization, although the all-cause mortality rate remained unchanged (113). Recently, the results of an internationally randomized trial, AFFIRM-AHF, were published, in which 1132 patients with acute HF and ID (using the criteria outlined above) were randomly assigned to i.v. ferric carboxymaltose (FCM) or placebo (114). Over a 52-week follow up, i.v. iron did not improve mortality, while it was associated with lower risk of hospitalization and a slight increase ( 0.5 $\mathrm{mg} / \mathrm{dL}$ ) in hemoglobin (114). A subanalysis of the data showed that the benefit was observed in patients with FTN less than 100 $\mathrm{ng} / \mathrm{mL}$ and not in those with FTN between 100 and $300 \mathrm{ng} /$ $\mathrm{mL}$, suggesting that iron supplementation benefits only patients who are truly ID. Additionally, increases in the labile pool of iron within cardiomyocytes can directly contribute to cardiomyopathy via lipid peroxidation and induction of ferroptosis, and fol- lowing pressure overload, ferritinophagy is augmented, resulting in the mobilization of iron stores and an increase in the pool of labile iron (115). As i.v. iron delivers large amounts of iron to tissues, including cardiomyocytes, the potential for i.v. iron to exacerbate cardiomyocyte damage in the setting of cardiac injury in humans requires further evaluation.

The efficacy of oral versus i.v. iron in HF has not been studied adequately. The IRON-HF study was a trial of 23 patients with ID (FTN $<500 \mathrm{ng} / \mathrm{mL}$ and TSAT $<20 \%$ ) to compare oral and i.v. supplementations, but the study was terminated for lack of funding (116). Analysis of the data from this study revealed improved maximal oxygen consumption achieved during exercise $\left(\mathrm{VO}_{2} \max \right)$ in the i.v. iron group and increased FTN and TSAT in both i.v. and oral groups. Another randomized clinical trial (IRONOUT-HF) compared the efficacy of oral iron polysaccharide versus placebo in $\mathrm{HF}$ patients with reduced ejection fraction $(<40 \%)$ and FTN between 15 and $100 \mathrm{ng} / \mathrm{mL}$, or FTN between 100 and $299 \mathrm{ng} / \mathrm{mL}$ with TSAT less than $20 \%$. The results of this study showed that 16 weeks of $150 \mathrm{mg}$ twice daily of oral iron increased FTN and TSAT levels, while $\mathrm{VO}_{2}$ max, 6-minute walk distance, health status, and plasma N-terminal pro-B-type natriuretic peptide (pro-BNP) were not different between the treatment groups (117). Importantly, the increase in FTN and TSAT was inversely correlated with the baseline hepcidin levels, as patients with lower hepcidin benefited most from oral iron supplementation (117). These results indicate that HF patients with true ID would still benefit from oral iron supplementation. Recently, a prospective study showed that 3-month therapy with low-dose oral sucrosomial iron in HF patients with ID increased iron indices and improved exercise capacity and quality of life up to 6 months. There was also a trend toward reduced BNP with oral sucrosomial iron (118). Large clinical trials to evaluate effectiveness and safety profiling of oral versus i.v. iron formulations in $\mathrm{HF}$ patients are thus necessary.

In contrast to current advocacy to treat HF patients with i.v. iron supplementation, there is evidence that certain patients with coronary artery disease may benefit from chelation therapy. The Trial to Assess Chelation Therapy (TACT) was designed to assess the effects of EDTA (a divalent anion chelator) in patients with stable coronary artery disease at least 6 months after myocardial infarction (MI) (119). Patients received 40 infusions of $500 \mathrm{~mL}$ chelation (3 $\mathrm{g}$ of disodium EDTA, $7 \mathrm{~g}$ of ascorbate, B vitamins, electrolytes, procaine, and heparin) or placebo. Although no effect on all-cause mortality was observed, the chelation therapy was associated with a significant decrease in the composite of mortality, MI, stroke, coronary artery revascularization, and hospitalization due to angina. Importantly, two subgroups of patients, diabetes mellitus and anterior MI, showed a robust reduction in cardiovascular events and benefited more from chelation therapy (119). It should be noted that EDTA binds many different divalent and trivalent cations, and the treatment in the TACT trial contained other chemical components, as indicated above. A larger study called TACT-II is currently being conducted to confirm the results from the TACT trial (120).

\section{Cancer}

Disordered iron homeostasis and anemia are common features of both hematopoietic and solid malignancy (121). Cancer-asso- 
ciated anemia is correlated with poor prognosis and reduction in quality-of-life measures, making the correction of anemia a clinical priority (122). The precise clinical cutoffs that define absolute ID in cancer can vary, but TSAT less than $20 \%$ and serum FTN less than $100 \mathrm{ng} / \mathrm{mL}$ have been proposed (123).

Cancer cells depend on iron to maintain anabolic processes and support energy metabolism. Populations with high iron levels, such as individuals who receive frequent blood transfusions or have diseases of iron overload such as hemochromatosis, are at greater risk of developing cancer over their lifetime $(124,125)$. Conversely, individuals who undergo phlebotomy regularly have lower incidences of cancer (126). Iron is required for protein translation, nucleotide biosynthesis, oxidative metabolism, and DNA and histone demethylation. Therefore, the exploitation of iron metabolism, either through iron chelation or sensitizing of cells to iron-mediated cell death (ferroptosis), has received much interest as adjuvant treatment for malignancy.

Clinically available iron chelators such as deferoxamine (DFO) have been shown to prevent cancer cell proliferation and induce apoptosis in multiple cancer types (127). Perhaps one of the clearest examples of a cancer cell's dependence on iron is in leptomeningeal metastases (LMs). LM cells have been shown to secrete high levels of lipocalin-2 (LCN2), a protein that can scavenge iron from the extracellular environment. Both reduction in LCN2 and iron chelation with DFO significantly reduced tumor burden and improved survival in an in vivo model of LM (128). By contrast, many cancer types have been shown to be sensitive to inducers of ferroptosis, a form of cell death in which ROS generated by excess iron or glutathione deficiency causes lipid peroxidation and cell death $(129,130)$.

The treatment of ID with iron in the setting of malignancy is complicated by the differential effects of iron on erythropoiesis versus the malignancy itself (131). There is little debate that significant ID anemia requires correction, but the safety of iron therapy in cancer is not clear $(123,132,133)$. Therefore, there is considerable need to (a) identify methods of restricting iron replacement to the bone marrow to support erythropoiesis, and (b) identify biomarkers of tumors with significant iron demand that could serve to identify subpopulations at risk for worsening disease if given i.v. iron supplementation.

\section{Oral versus i.v. iron supplementation}

A number of i.v. and oral iron replacement products are available in the US market. Oral iron supplementation is the most common form of therapy for ID, is usually well tolerated, and is associated with proper response to the treatment (88). There are several formulations of oral iron, including ferrous fumarate, ferrous gluconate, and ferrous sulfate (134). The limiting factors with oral supplementation that can lead to treatment noncompliance are the gastrointestinal (GI) side effects, including metallic taste, nausea, vomiting, bloating, constipation, and GI upset. Poor GI absorption is also reported in patients with chronic inflammation and celiac and Crohn's disease (135). Oral iron supplementation may also be associated with altered gut microbiome composition, which is more often seen in a pathogenic state (136). Newer oral iron formulations, such as ferric maltol, are commonly associated with superior absorption and tolerability $(137,138)$.
Low-molecular weight iron dextran, ferric gluconate, iron sucrose, ferumoxytol, iron isomaltoside, and ferric carboxymaltose (FCM) are among the commercially available forms of i.v. iron. Intravenous iron preparations provide large amounts of iron into the circulation in a short time; however, they also bypass the physiological pathways of iron absorption and regulation in the GI tract. Earlier forms of i.v. iron, like iron dextran, were associated with large increases in the labile iron pool in circulation and ROS production (111). However, new formulations, like FCM, iron sucrose, and ferumoxytol, are designed with larger and more stable carbohydrate shells and release lower levels of free iron into the circulation, resulting in fewer side effects (139). In recent years, several reports have indicated that i.v. iron (especially FCM) can lead to hypophosphatemia, due to elevated levels of FGF23 $(140,141)$. Some patients with this side effect have required hip replacement surgery (142).

The FDA drug label for FCM states that the therapy is indicated "for the treatment of iron deficiency anemia in adult patients who have intolerance to oral iron or have had an unsatisfactory response to oral iron [or who have] non-dialysis dependent chronic kidney disease." Thus, patients with uncomplicated ID should always be considered for oral iron formulations, given the ease of administration, fewer side effects, and cost-effectiveness, before i.v. iron therapy is instituted. Intravenous iron should only be considered if oral iron cannot be tolerated and in non-dialysis-dependent CKD patients.

\section{Conclusions}

Due to absorbable ferrous iron's scarcity in the environment, mammals have evolved a sophisticated system to absorb iron from the GI tract and maintain sufficient iron stores. Dietary ferric iron is first reduced to the ferrous form and is then absorbed by duodenal DMT1 and transported into the circulation via FPN1. In addition, macrophages export iron recycled from senescent RBCs through FPN1. Systemic iron levels are regulated by hepcidin, hypoxia, and inflammation. In the circulation, iron is bound to TF, and is uptaken by cells expressing TFR1 via receptor-mediated endocytosis. Intracellular iron can be found incorporated into heme and Fe-S clusters or bound to proteins, such as FTN, to prevent iron toxicity.

ID is predominantly associated with anemia and a number of other disorders that occur mostly during development. However, in certain patients with chronic disease, ID diagnosis can be challenging, because inflammation causes reduced systemic iron availability. Iron supplementation should be reserved only for those who are truly iron deficient, as excess iron supplementation can put patients at risk for unwanted side effects. Intravenous iron supplementation should only be considered in certain groups of patients who are intolerant of oral iron and patients with CKD. Finally, at present, the clinical data are insufficient regarding the long-term safety and efficacy of i.v. iron.

\section{Acknowledgments}

HA is supported by NIH grants NHLBI HL140973, HL138982, and HL140927, and the Leducq Foundation.

Address correspondence to: Hossein Ardehali, SQBRC 8-521, 303 E. Superior Avenue, Chicago, Illinois 60611, USA. Phone: 312.503.2342; Email: h-ardehali@northwestern.edu. 
1. Wallace DF. The regulation of iron absorption and homeostasis. Clin Biochem Rev. 2016;37(2):51-62.

2. Meng $\mathrm{Y}$, et al. Jumonji domain-containing protein family: the functions beyond lysine demethylation. J Mol Cell Biol. 2018;10(4):371-373.

3. Shen J, et al. Structure and mechanism of a unique diiron center in mammalian Stearoyl-CoA desaturase. J Mol Biol. 2020;432(18):5152-5161.

4. Braymer JJ, Lill R. Iron-sulfur cluster biogenesis and trafficking in mitochondria.J Biol Chem. 2017;292(31):12754-12763.

5. Ganz T. Systemic iron homeostasis. Physiol Rev. 2013;93(4):1721-1741.

6. Winterbourn CC. Toxicity of iron and hydrogen peroxide: the Fenton reaction. Toxicol Lett. 1995;82-83:969-974.

7. Cerami C. Iron nutriture of the fetus, neonate, infant, and child. Ann Nutr Metab. 2017;71(suppl 3):8-14.

8. Inoue $\mathrm{K}$, et al. Functional characterization of PCFT/HCP1 as the molecular entity of the carrier-mediated intestinal folate transport system in the rat model. Am J Physiol Gastrointest Liver Physiol. 2008;294(3):G660-G668.

9. Przybyszewska J, Zekanowska E. The role of hepcidin, ferroportin, HCP1, and DMT1 protein in iron absorption in the human digestive tract. Prz Gastroenterol. 2014;9(4):208-213.

10. West AR, Oates PS. Mechanisms of heme iron absorption: current questions and controversies. World J Gastroenterol. 2008;14(26):4101-4110.

11. Hungerford DM Jr., Linder MC. Interactions of $\mathrm{pH}$ and ascorbate in intestinal iron absorption. J Nutr. 1983;113(12):2615-2622.

12. Lane DJ, et al. Duodenal cytochrome b (DCYTB) in iron metabolism: an update on function and regulation. Nutrients. 2015;7(4):2274-2296.

13. Briguglio $\mathrm{M}$, et al. The central role of iron in human nutrition: from folk to contemporary medicine. Nutrients. 2020;12(6):1761

14. Shawki A, et al. Intestinal DMT1 is critical for iron absorption in the mouse but is not required for the absorption of copper or manganese. Am J Physiol Gastrointest Liver Physiol. 2015;309(8):G635-G647.

15. Manatschal C, et al. Mechanistic basis of the inhibition of SLC11/NRAMP-mediated metal ion transport by bis-isothiourea substituted compounds. Elife. 2019;8:e51913.

16. Gao G, et al. Cellular iron metabolism and regulation. Adv Exp Med Biol. 2019;1173:21-32.

17. Donovan A, et al. The iron exporter ferroportin/ Slc40a1 is essential for iron homeostasis. Cell Metab. 2005;1(3):191-200.

18. Kaplan J, Ward DM. The essential nature of iron usage and regulation. Curr Biol. 2013;23(15):R642-R646.

19. Cherukuri S, et al. Unexpected role of ceruloplasmin in intestinal iron absorption. Cell Metab. 2005;2(5):309-319.

20. Ponka P. Cellular iron metabolism. Kidney Int Suppl. 1999;69:S2-S11.

21. Billesbolle CB, et al. Structure of hepcidin-bound ferroportin reveals iron homeostatic mechanisms. Nature. 2020;586(7831):807-811.

22. Sharp P, Srai SK. Molecular mechanisms involved in intestinal iron absorption. World J Gastroenterol. 2007;13(35):4716-4724.

23. Khandelwal S, et al. Reduced expression of CD47 during murine red blood cell (RBC) senescence and its role in RBC clearance from the circulation. Transfusion. 2007;47(9):1725-1732.

24. Oldenborg PA, et al. Role of CD47 as a marker of self on red blood cells. Science. 2000;288(5473):2051-2054.

25. Sukhbaatar N, Weichhart T. Iron regulation: macrophages in control. Pharmaceuticals (Basel). 2018;11(4):137.

26. White C, et al. HRG1 is essential for heme transport from the phagolysosome of macrophages during erythrophagocytosis. Cell Metab. 2013;17(2):261-270.

27. Liu Q, et al. Hypoxia-inducible factor regulates hepcidin via erythropoietin-induced erythropoiesis. JClin Invest. 2012;122(12):4635-4644.

28. Wang CY, Babitt JL. Hepcidin regulation in the anemia of inflammation. Curr Opin Hematol. 2016;23(3):189-197.

29. Ganz T. Hepcidin and iron regulation, 10 years later. Blood. 2011:117(17):4425-4433.

30. Babitt JL, et al. Bone morphogenetic protein signaling by hemojuvelin regulates hepcidin expression. Nat Genet. 2006;38(5):531-539.

31. Andriopoulos B Jr., et al. BMP6 is a key endogenous regulator of hepcidin expression and iron metabolism. Nat Genet. 2009;41(4):482-487.

32. Canali S, et al. Endothelial cells produce bone morphogenetic protein 6 required for iron homeostasis in mice. Blood. 2017;129(4):405-414.

33. Canali S, et al. Bone morphogenetic protein 2 controls iron homeostasis in mice independent of Bmp6. Am J Hematol. 2017;92(11):1204-1213.

34. Lim PJ, et al. Nrf2 controls iron homeostasis in haemochromatosis and thalassaemia via Bmp6 and hepcidin. Nat Metab. 2019;1(5):519-531.

35. Kautz L, et al. Identification of erythroferrone as an erythroid regulator of iron metabolism. Nat Genet. 2014;46(7):678-684

36. Srole DN, Ganz T. Erythroferrone structure, function, and physiology: iron homeostasis and beyond. J Cell Physiol. 2021;236(7):4888-4901.

37. Babitt JL, Lin HY. Molecular mechanisms of hepcidin regulation: implications for the anemia of CKD. Am J Kidney Dis. 2010;55(4):726-741.

38. Roetto A, et al. Mutant antimicrobial peptide hepcidin is associated with severe juvenile hemochromatosis. Nat Genet. 2003;33(1):21-22.

39. Kong X, et al. Genotypic and phenotypic spectra of hemojuvelin mutations in primary hemochromatosis patients: a systematic review. Orphanet $J$ Rare Dis. 2019;14(1):171.

40. Kowdley KV, et al. ACG clinical guideline: hereditary hemochromatosis. Am J Gastroenterol. 2019;114(8):1202-1218.

41. Shah YM, Xie L. Hypoxia-inducible factors link iron homeostasis and erythropoiesis. Gastroenterology. 2014;146(3):630-642.

42. Rankin EB, et al. Hypoxia-inducible factor-2 (HIF-2) regulates hepatic erythropoietin in vivo. JClin Invest. 2007;117(4):1068-1077.

43. Shah YM, et al. Intestinal hypoxia-inducible transcription factors are essential for iron absorption following iron deficiency. Cell Metab. 2009;9(2):152-164.
44. Drakesmith H, Prentice AM. Hepcidin and the iron-infection axis. Science. 2012;338(6108):768-772.

45. Ganz T, Nemeth E. Iron homeostasis in host defence and inflammation. Nat Rev Immunol. 2015;15(8):500-510.

46. Weiss G, et al. Anemia of inflammation. Blood. 2019;133(1):40-50.

47. Mayeur C, et al. Oral administration of a bone morphogenetic protein type I receptor inhibitor prevents the development of anemia of inflammation. Haematologica. 2015;100(2):e68-e71.

48. Fung E, Nemeth E. Manipulation of the hepcidin pathway for therapeutic purposes. Haematologica. 2013;98(11):1667-1676.

49. Luck AN, Mason AB. Transferrin-mediated cellular iron delivery. Curr Top Membr. 2012;69:3-35.

50. Mancias JD, et al. Quantitative proteomics identifies NCOA4 as the cargo receptor mediating ferritinophagy. Nature. 2014;509(7498):105-109.

51. Quiles Del Rey M, Mancias JD. NCOA4-mediated ferritinophagy: a potential link to neurodegeneration. Front Neurosci. 2019;13:238

52. Yambire KF, et al. Impaired lysosomal acidification triggers iron deficiency and inflammation in vivo. Elife. 2019;8:e51031.

53. Richardson DR, et al. Mitochondrial iron trafficking and the integration of iron metabolism between the mitochondrion and cytosol. Proc Natl Acad Sci U S A. 2010;107(24):10775-10782.

54. Shaw GC, et al. Mitoferrin is essential for erythroid iron assimilation. Nature. 2006;440(7080):96-100.

55. Troadec MB, et al. Targeted deletion of the mouse Mitoferrin 1 gene: from anemia to protoporphyria. Blood. 2011;117(20):5494-5502.

56. Seguin A, et al. The mitochondrial metal transporters mitoferrin 1 and mitoferrin 2 are required for liver regeneration and cell proliferation in mice. J Biol Chem. 2020;295(32):11002-11020.

57. Corsi B, et al. Human mitochondrial ferritin expressed in HeLa cells incorporates iron and affects cellular iron metabolism. J Biol Chem. 2002;277(25):22430-22437.

58. Ichikawa Y, et al. Disruption of ATP-binding cassette B8 in mice leads to cardiomyopathy through a decrease in mitochondrial iron export. Proc Natl Acad Sci U S A. 2012;109(11):4152-4157.

59. Pearson SA, Cowan JA. Evolution of the human mitochondrial ABCB7 [2Fe-2S] (GS) ${ }_{4}$ cluster exporter and the molecular mechanism of an E433K disease-causing mutation. Arch Biochem Biophys. 2021;697:108661.

60. Bayeva M, et al. ATP-binding cassette B10 regulates early steps of heme synthesis. Circ Res. 2013;113(3):279-287.

61. Rouault TA. The role of iron regulatory proteins in mammalian iron homeostasis and disease. Nat Chem Biol. 2006;2(8):406-414.

62. Bayeva M, et al. When less is more: novel mechanisms of iron conservation. Trends Endocrinol Metab. 2013;24(11):569-577.

63. Bayeva $\mathrm{M}$, et al. mTOR regulates cellular iron homeostasis through tristetraprolin. Cell Metab. 2012;16(5):645-657.

64. Sandler H, et al. Not1 mediates recruitment of the deadenylase Caf1 to mRNAs targeted for degradation by tristetraprolin. Nucleic Acids Res. 
2011;39(10):4373-4386.

65. Sato T, et al. mRNA-binding protein tristetraprolin is essential for cardiac response to iron deficiency by regulating mitochondrial function. Proc Natl Acad Sci U S A. 2018;115(27):E6291-E6300.

66. Camaschella C. Iron deficiency: new insights into diagnosis and treatment. Hematology Am Soc Hematol Educ Program. 2015;2015:8-13.

67. Muñoz M, et al. Current misconceptions in diagnosis and management of iron deficiency. Blood Transfus. 2017;15(5):422-437.

68. Philip KEJ, et al. The prevalence and associated mortality of non-anaemic iron deficiency in older adults: a 14 years observational cohort study. $\mathrm{Br} \mathrm{J}$ Haematol. 2020;189(3):566-572.

69. Camaschella C. Iron-deficiency anemia. $N$ Engl J Med. 2015;372(19):1832-1843.

70. Ren A, et al. Low first-trimester hemoglobin and low birth weight, preterm birth and small for gestational age newborns. Int J Gynaecol Obstet. 2007;98(2):124-128.

71. Ceppi A, Blum JW. Effects of growth hormone on growth performance, haematology, metabolites and hormones in iron-deficient veal calves. Zentralbl Veterinarmed A. 1994;41(6):443-458.

72. Medeiros DM, et al. Iron deficiency negatively affects vertebrae and femurs of rats independently of energy intake and body weight. J Nutr. 2004;134(11):3061-3067.

73. Georgieff MK, et al. Nutritional influences on brain development. Acta Paediatr. 2018;107(8):1310-1321.

74. Todorich B, et al. Oligodendrocytes and myelination: the role of iron. Glia. 2009;57(5):467-478.

75. Amin SB, et al. In utero iron status and auditory neural maturation in premature infants as evaluated by auditory brainstem response. J Pediatr. 2010;156(3):377-381.

76. Armony-Sivan R, et al. Iron status and neurobehavioral development of premature infants. JPerinatol. 2004;24(12):757-762.

77. Dallman PR. Iron restriction in the nursing rat: early effects upon tissue heme proteins, hemoglobin and liver iron. J Nutr. 1969;97(4):475-480.

78. Matak P, et al. Disrupted iron homeostasis causes dopaminergic neurodegeneration in mice. Proc Natl Acad Sci U S A. 2016;113(13):3428-3435.

79. Bastian TW, et al. The effects of early-life iron deficiency on brain energy metabolism. Neurosci Insights. 2020;15:2633105520935104.

80. Carlson ES, et al. Perinatal iron deficiency results in altered developmental expression of genes mediating energy metabolism and neuronal morphogenesis in hippocampus. Hippocampus. 2007;17(8):679-691.

81. Jayaweera J, et al. Childhood iron deficiency anemia leads to recurrent respiratory tract infections and gastroenteritis. Sci Rep. 2019;9(1):12637.

82. de Silva A, et al. Iron supplementation improves iron status and reduces morbidity in children with or without upper respiratory tract infections: a randomized controlled study in Colombo, Sri Lanka. Am J Clin Nutr. 2003;77(1):234-241.

83. Stoffel NU, et al. Iron deficiency anemia at time of vaccination predicts decreased vaccine response and iron supplementation at time of vaccination increases humoral vaccine response: a birth cohort study and a randomized trial fol- low-up study in Kenyan infants. Front Immunol. 2020;11:1313.

84. Kuvibidila SR, et al. Iron deficiency reduces serum and in vitro secretion of interleukin- 4 in mice independent of altered spleen cell proliferation. Nutr Res. 2012;32(2):107-115.

85. Kuvibidila SR, et al. Iron deficiency, but not underfeeding reduces the secretion of interferon-gamma by mitogen-activated murine spleen cells. Cytokine. 2010;52(3):230-237.

86. Aly SS, et al. Assessment of peripheral blood lymphocyte subsets in children with iron deficiency anemia. BMC Pediatr. 2018;18(1):49.

87. Mullick S, et al. Impact of iron deficiency anaemia on T lymphocytes \& their subsets in children. Indian JMed Res. 2006;124(6):647-654.

88. Jonker FA, Boele van Hensbroek M. Anaemia, iron deficiency and susceptibility to infections. J Infect. 2014;69(suppl 1):S23-S27.

89. Accari SL, Fisher PR. Emerging roles of JmjC domain-containing proteins. Int Rev Cell Mol Biol. 2015;319:165-220.

90. Jiang Y, et al. Iron-dependent histone 3 lysine 9 demethylation controls B cell proliferation and humoral immune responses. Nat Commun 2019;10(1):2935.

91. Wang Z, et al. Iron drives T helper cell pathogenicity by promoting RNA-binding protein PCBP1-mediated proinflammatory cytokine production. Immunity. 2018;49(1):80-92.

92. Ohayon MM, et al. Epidemiology of restless legs syndrome: a synthesis of the literature. Sleep Med Rev. 2012;16(4):283-295.

93. Li G, et al. Association of BTBD9 and MAP2K5/ SKOR1 with restless legs syndrome in Chinese population. Sleep. 2017;40(4):zsx028.

94. El Gewely M, et al. Reassessing GWAS findings for the shared genetic basis of insomnia and restless legs syndrome. Sleep. 2018;41(11):zsy164.

95. Rizzo G, et al. Low brain iron content in idiopathic restless legs syndrome patients detected by phase imaging. Mov Disord. 2013;28(13):1886-1890.

96. Memon MD, et al. Unraveling the mysteries of restless leg syndrome. Cureus. 2020;12(10):e10951.

97. Winkelman JW, et al. Practice guideline summary: Treatment of restless legs syndrome in adults: report of the Guideline Development, Dissemination, and Implementation Subcommittee of the American Academy of Neurology. Neurology. 2016;87(24):2585-2593.

98. Shaikh H, Aeddula NR. Anemia of chronic renal disease. In: StatPearls. StatPearls Publishing; 2020. https://www.ncbi.nlm.nih.gov/books/ NBK539871/. Accessed April 13, 2021.

99. Wojtaszek E, et al. Iron and chronic kidney disease: still a challenge. Front Med (Lausanne). 2020;7:565135.

100.Moist LM, et al. Canadian Society of Nephrology commentary on the 2012 KDIGO Clinical Practice Guideline for Anemia in CKD. Am J Kidney Dis. 2013;62(5):860-873.

101.Cappellini MD, et al. Iron deficiency across chronic inflammatory conditions: international expert opinion on definition, diagnosis, and management. Am J Hematol. 2017;92(10):1068-1078. 102. Madore F, et al. Clinical practice guidelines for assessment and management of iron deficiency.
Kidney Int Suppl. 2008(110):S7-S11.

103. McMurray JJ, et al. Kidney disease: improving global outcomes (KDIGO) anemia work group. KDIGO clinical practice guideline for anemia in chronic kidney disease. Kidney Int Suppl. 2012;2(4):279-335.

104. O'Lone EL, et al. Parenteral versus oral iron therapy for adults and children with chronic kidney disease. Cochrane Database Syst Rev. 2019;2(2):Cd007857.

105. Pergola PE, et al. Ziltivekimab for treatment of anemia of inflammation in patients on hemodialysis: results from a phase $1 / 2$ multicenter, randomized, double-blind, placebo-controlled trial JAm Soc Nephrol. 2021;32(1):211-222.

106.Ponikowski P, et al. 2016 ESC Guidelines for the diagnosis and treatment of acute and chronic heart failure: the Task Force for the diagnosis and treatment of acute and chronic heart failure of the European Society of Cardiology (ESC). Developed with the special contribution of the Heart Failure Association (HFA) of the ESC. Eur Heart J. 2016;37(27):2129-2200.

107. Yancy CW, et al. 2017 ACC/AHA/HFSA focused update of the 2013 ACCF/AHA Guideline for the Management of Heart Failure: a report of the American College of Cardiology/American Heart Association Task Force on Clinical Practice Guidelines and the Heart Failure Society of America. JCard Fail. 2017;23(8):628-651.

108. Jankowska EA, et al. Iron status in patients with chronic heart failure. Eur Heart J. 2013;34(11):827-834.

109. Divakaran V, et al. Hepcidin in anemia of chronic heart failure. Am J Hematol. 2011;86(1):107-109.

110. Punj S, et al. Iron deficiency and supplementation in heart failure and chronic kidney disease. $\mathrm{Mol}$ Aspects Med. 2020;75:100873.

111. Ghafourian K, et al. Iron and heart failure: diagnosis, therapies, and future directions. JACC Basic Transl Sci. 2020;5(3):300-313.

112. Ghafourian K, et al. Intravenous iron therapy in heart failure: a different perspective. Eur J Heart Fail. 2019;21(6):703-714.

113. Zhou X, et al. Iron supplementation improves cardiovascular outcomes in patients with heart failure. Am JMed. 2019;132(8):955-963.

114. Ponikowski P, et al. Ferric carboxymaltose for iron deficiency at discharge after acute heart failure: a multicentre, double-blind, randomised, controlled trial. Lancet. 2020;396(10266):1895-1904.

115. Ito J, et al. Iron derived from autophagymediated ferritin degradation induces cardiomyocyte death and heart failure in mice. Elife. 2021;10:e62174.

116. Beck-da-Silva L, et al. IRON-HF study: a randomized trial to assess the effects of iron in heart failure patients with anemia. Int J Cardiol. 2013;168(4):3439-3442.

117. Lewis GD, et al. Effect of oral iron repletion on exercise capacity in patients with heart failure with reduced ejection fraction and iron deficiency: the IRONOUT HF Randomized Clinical Trial. JAMA. 2017;317(19):1958-1966.

118. Karavidas A, et al. Oral sucrosomial iron improves exercise capacity and quality of life in heart failure with reduced ejection fraction and iron deficiency: a non-randomized, open- 
label, proof-of-concept study [published online January 9, 2021]. Eur J Heart Fail. https://doi. org/10.1002/ejhf.2092.

119. Lamas GA, et al. Effect of disodium EDTA chelation regimen on cardiovascular events in patients with previous myocardial infarction: the TACT randomized trial. JAMA. 2013;309(12):1241-1250.

120. Lamas GA, et al. Heavy metals, cardiovascular disease, and the unexpected benefits of chelation therapy. J Am Coll Cardiol. 2016;67(20):2411-2418.

121. Ludwig H, et al. The European Cancer Anaemia Survey (ECAS): a large, multinational, prospective survey defining the prevalence, incidence, and treatment of anaemia in cancer patients. Eur JCancer. 2004;40(15):2293-2306.

122. Harper P, Littlewood T. Anaemia of cancer: impact on patient fatigue and long-term outcome. Oncology. 2005;69(suppl 2):2-7.

123. Aapro M, et al. Management of anaemia and iron deficiency in patients with cancer: ESMO Clinical Practice Guidelines. Ann Oncol. 2018;29(suppl 4):iv96-iv110.

124. Elmberg $\mathrm{M}$, et al. Cancer risk in patients with hereditary hemochromatosis and in their first-degree relatives. Gastroenterology. 2003;125(6):1733-1741.

125. Hjalgrim $\mathrm{H}$, et al. Cancer incidence in blood transfusion recipients. J Natl Cancer Inst.
2007;99(24):1864-1874.

126. Merk K, et al. The incidence of cancer among blood donors. Int JEpidemiol. 1990;19(3):505-509.

127. Hsu MY, et al. Iron: an essential element of cancer metabolism. Cells. 2020;9(12):2591.

128. Chi Y, et al. Cancer cells deploy lipocalin-2 to collect limiting iron in leptomeningeal metastasis. Science. 2020;369(6501):276-282.

129. Dixon SJ, et al. Ferroptosis: an iron-dependent form of nonapoptotic cell death. Cell. 2012;149(5):1060-1072.

130. Viswanathan VS, et al. Dependency of a therapy-resistant state of cancer cells on a lipid peroxidase pathway. Nature. 2017;547(7664):453-457.

131. Manz DH, et al. Iron and cancer: recent insights. Ann N Y Acad Sci. 2016;1368(1):149-161.

132. Gilreath JA, et al. Total dose iron dextran infusion in cancer patients: is it SaFe2+? J Natl Compr Canc Netw. 2012;10(5):669-676.

133. Busti F, et al. Anemia and iron deficiency in cancer patients: role of iron replacement therapy. Pharmaceuticals (Basel). 2018;11(4):94.

134. Gasche $\mathrm{C}$, et al. Ferric maltol is effective in correcting iron deficiency anemia in patients with inflammatory bowel disease: results from a phase-3 clinical trial program. Inflamm Bowel Dis. 2015;21(3):579-588.

135. Bayraktar UD, Bayraktar S. Treatment of iron deficiency anemia associated with gastroin- testinal tract diseases. World J Gastroenterol. 2010;16(22):2720-2725.

136. Mahalhal A, et al. Oral iron exacerbates colitis and influences the intestinal microbiome. PLOS One. 2018;13(10):e0202460.

137. Khoury A, et al. Ferric maltol: a new oral iron formulation for the treatment of iron deficiency in adults. Ann Pharmacother. 2021;55(2):222-229.

138. Tanaka M, et al. Effect of a ferric citrate formulation, a phosphate binder, on oxidative stress in chronic kidney diseases-mineral and bone disorder patients receiving hemodialysis: a pilot study. Biol Pharm Bull. 2016;39(6):1000-1006.

139. Auerbach M, Macdougall I. The available intravenous iron formulations: history, efficacy, and toxicology. Hemodial Int. 2017;21(suppl 1):S83-S92.

140.Teh KK, et al. Severe symptomatic hypophosphataemia as a complication of parenteral iron replacement. Eur JCase Rep Intern Med. 2020;7(11):001860.

141. Schaefer B, et al. Hypophosphataemia after treatment of iron deficiency with intravenous ferric carboxymaltose or iron isomaltoside-a systematic review and meta-analysis [published online November 13, 2020]. Br JClin Pharmacol. https:// doi.org/10.1111/bcp.14643.

142.Tozzi D, Tozzi J. Osteomalacia and insufficiency fractures secondary to intravenous iron therapy: a case report. J Orthop Case Rep. 2020;10(1):4-7. 